\title{
Design and implementation of a secure and user-friendly broker platform supporting the end-to-end provisioning of e-homecare services.
}

\author{
S. Van Hoecke, K. Steurbaut, K. Taveirne, F. De Turck, B. Dhoedt. \\ Department of Information Technology (INTEC), Ghent University - IBCN - IBBT
}

\section{Summary}

Because of growing financial pressures in healthcare and a shift in pathologies, patients are discharged earlier and care is organized at home, requiring e-homecare services to support care and patient integration in society. As these services are built by different vendors, integration is complex.

Therefore a broker platform has been designed using the web service technology. The broker allows efficient data communication and guarantees quality requirements such as security, availability and cost-efficiency by dynamically selecting and composing services, minimizing user interactions and simplifying authentication through single-signon so that users do not need to authenticate to each service separately.

A platform prototype and several e-homecare services (e-alarm, tele-monitoring, audiodiary and video-chat) have been implemented and were evaluated by diabetes and multiple sclerosis patients. When a user starts the application, he is requested to authenticate himself using his electronic identity card. Once authenticated, a set of buttons is added to the client, giving access to the e-homecare services.

The startup time and overhead imposed by the platform was experienced by the patients to be small enough. Combined with having all e-homecare services integrated in a single

client application, requiring only one login, resulted in a high quality of experience by the patients.

\author{
Correspondence to: \\ dr. ir. Sofie Van Hoecke \\ Ghent University \\ Department of Information Technology \\ Gaston Crommenlaan 8 bus 201 \\ B-9050 Gent, Belgium \\ Phone: +3293314940 \\ Fax: +3293314899 \\ E-mail: sofie.vanhoecke@intec.ugent.be
}




\section{Introduction}

As a result of the falling birthrate [1Error! Reference source not found.] and the increased life expectancy [2], the world's population is ageing. This introduces new challenges. Since chronic diseases primarily affect older adults, the prevalence of chronic diseases increases with an ageing population. The ageing population and a shift in the burden of illness from acute (infections and injury) to chronic conditions (asthma, epilepsy, heart disease, cancer, etc.) drive up health costs and create a generation of people living with long-term illness and disability.

Because of the growing financial pressure in healthcare and a shift in pathologies, care is organised "away" from medical institutions, requiring a greater role of the patients in the treatment and care of their (chronic) illness compared to inpatient settings. This way, homecare evolves to a more need-based approach, where applications not solely focus on medical purposes but also on care and integration in society. This patient-centered concept of bringing the care from the hospital to the patient at home is expected to result in cost reduction and improved quality of life [3].

In order to do so, the healthcare system must evolve to provide the support that both patients and healthcare providers require. E-homecare solutions have to connect patients with their healthcare team and provide tools to help patients to become more active and engaged in their own care.

Despite the proven benefits, e-homecare is not yet widely used in real-life medical or health situations. As technology becomes less expensive, more ubiquitous and more accessible to larger numbers of people, the spectrum of stand-alone e-homecare applications broadens. During the last few years, several public and private initiatives developed different applications, ranging from doctor mobility (remote access to medical data) to patient mobility (remote monitoring of vital signals, tele-consultation, mobile medical record, wearable medical sensors). E-homecare services are developed to monitor patient's health and body functions from a distance in order to save valuable healthcare service time. Unfortunately, existing e-health applications are designed in an ad-hoc way and most of the time used for discrete clinical activities, raising the question of when and how these fragmented e-health systems, used in different contexts, will become interoperable.

Integrating e-homecare services and applications is however a complex task since these services and applications are built by different vendors, using different data definitions and exchange standards. Additionally, e-homecare uses insecure computer networks [Error! Reference source not found.]. As a consequence, monitoring medical data or physiological parameters in a home environment and sending data to remote information systems puts requirements on security, responsibility, integrity, safety and ease-of-use. The connections must further guarantee the delivery of each message, because the nondelivery can cause important medical information to be lost [5]. 
In order to realize this interoperability, Quality of Service (QoS) broker platforms can be used to act as mediators between the users and services in order to enable efficient service management and service selection as quality requirements such as price and response time can influence the choice of service. For example, e-alarm services should receive higher priority than social services. Furthermore, service provisioning and user interaction should be as simple as possible.

\section{Methods}

Figure 1 presents the designed e-homecare broker architecture that enables integration of e-homecare services, crossing service providers, implementation languages and operating systems in order to simplify user interactions and relieve users from service and credential management. The broker platform allows efficient data communication and guarantees quality requirements such as security, availability and price by dynamically selecting and composing e-health services. The e-homecare broker architecture also simplifies service provisioning. Service providers only need to provide a Web service interface on their applications, register them transparently to the framework and the platform takes care of all the rest.

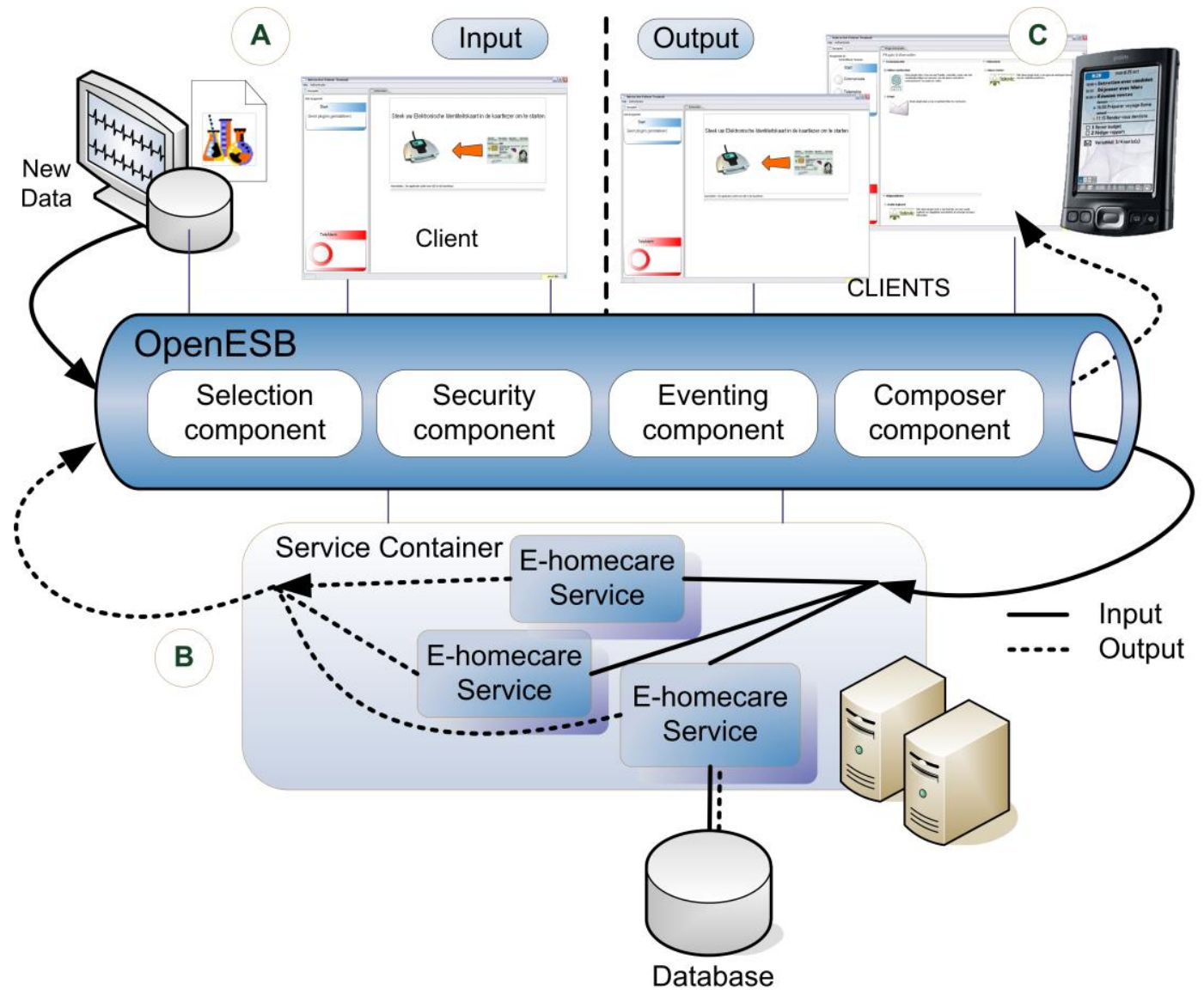

Figure 1: E-homecare broker platform: (A) events are pushed into the broker platform; (B) the appropriate services are activated for processing the data and generate new events or output data; (C) output data is sent to the clients. 
Whenever new data (e.g. medical data on a patient) is available, an event that contains this data is pushed into the broker platform (see Figure 1 part A). This data is provided automatically to all services which require this type of data as input (see Figure 1 part B). The services will process the data and generate new events or output data. This output can in turn be fed to other services or could be sent to the clients or the homecare nurse's PDA in case of an emergency (see Figure 1 part $C$ ).

The broker platform is implemented by extending the OpenESB messaging bus [6]. The components added to the ESB are a Selection component in order to provide failover and dynamic service selection [7], a Security component to provide a secure and safe architecture [8], an Eventing component in order to make the data delivery accurate and reliable [9], and finally a Composer component in order to create service orchestrations at runtime [10]. By enabling these components in the broker platform, users are relieved from service localization, user interactions are minimized and authentication is simplified by using single-sign-on.

As services can become unreachable by a number of circumstances or perform less well than guaranteed, the broker replaces at run-time the requested e-homecare service by another compatible service. By using dynamic endpoint binding, the broker can transparently switch between equivalent services, alleviating users from service localization and selection. On top of that, dynamic binding has the additional benefit that service providers can easily add new e-homecare services through a simple registration without the need to modify or complicate the broker process. The broker can use different selection algorithms to pick one of the available equivalent e-homecare services.

The remaining challenge is to create a client application as a single front-end to the platform, containing all the services offered by the platform and hiding from the users whether a simple or orchestrated service is concerned in order to simplify required user interactions. The client has to be very easy to use and needs to have a short time to learn.

Similar to the broker design that creates large applications as orchestrations of services, the same approach was taken for the client design. Therefore, a graphical subset of the Eclipse Rich Client Platform (RCP) [11] was created on which the e-homecare service providers can develop plug-ins. The plug-ins are in fact Open Standard Gateway Initiative (OSGi) bundles combined with the graphical features of the Eclipse IDE. Since OSGi is a dynamic component model, the plug-ins can be added to the client application without rebooting the application. As a consequence, by plugging in different modules at runtime, the client can be remotely managed and dynamically adjusted to the user profile.

As can be seen in Figure 2, when a user starts the application, he is requested to authenticate himself using his electronic identity card. Once authenticated, as presented in Figure 3, a set of buttons is added to the application, giving access to the patient specific modules and e-homecare services. 


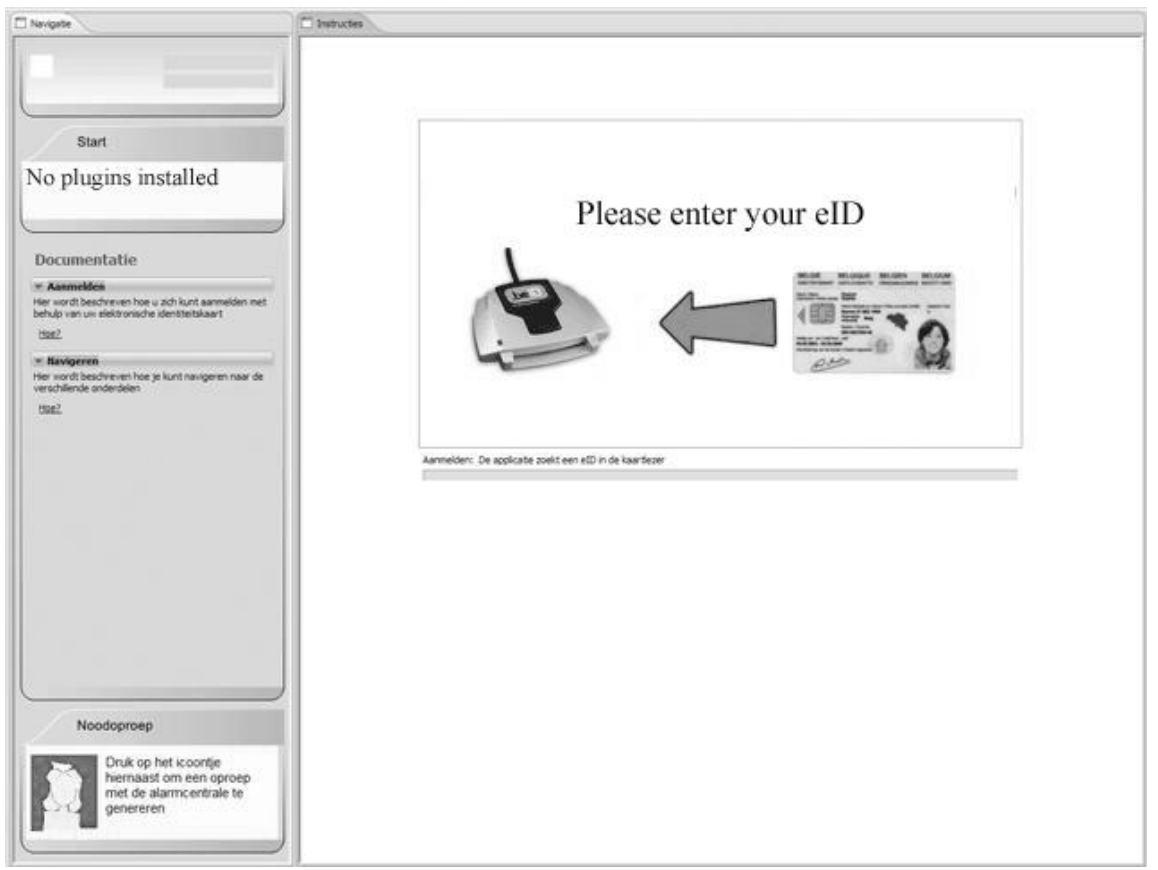

Figure 2: Client application using electronic identity card authentication.

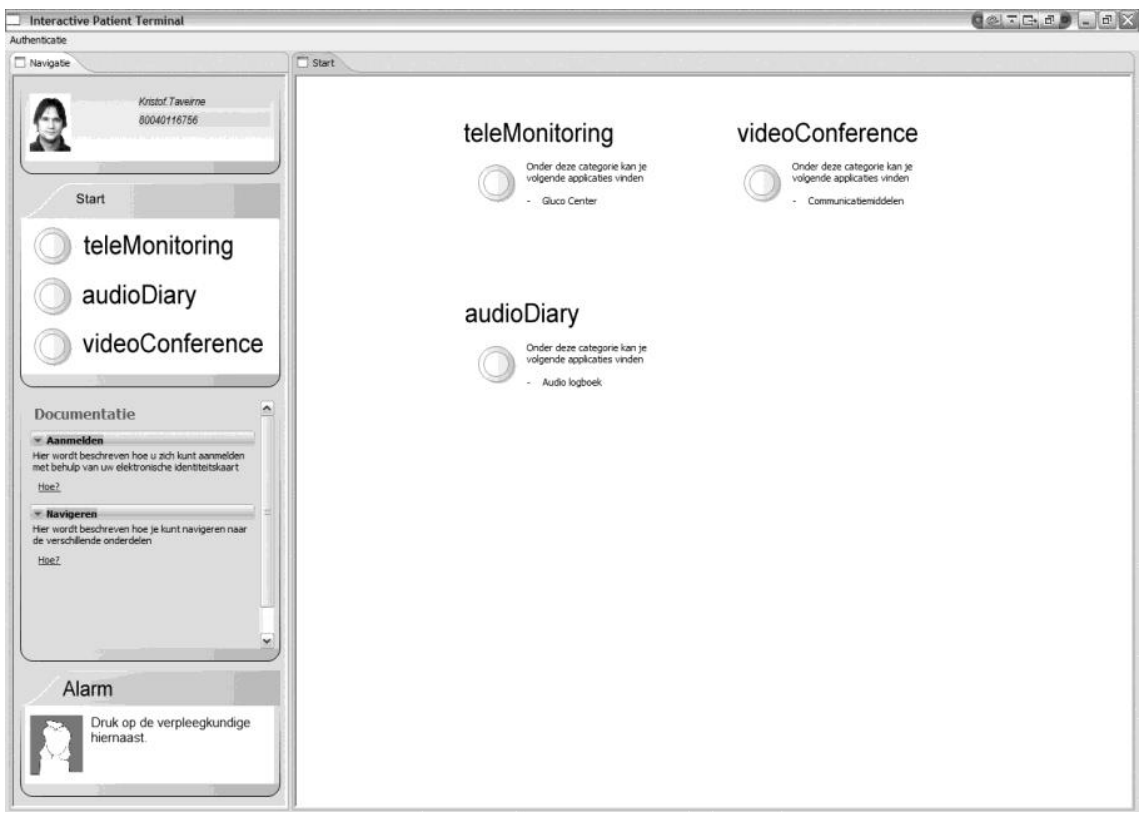

Figure 2: After logging in, buttons are added to the client giving access to the e-homecare services.

The e-homecare broker platform currently offers, but is not limited to, the following modules: e-scheduling, audio-diary, social games, video-chat, e-alarm, and telemonitoring (see Figure 3). Since each of the e-homecare service providers requires its own authentication, single-sign-on is used to relieve users from authenticating to each individual service. 


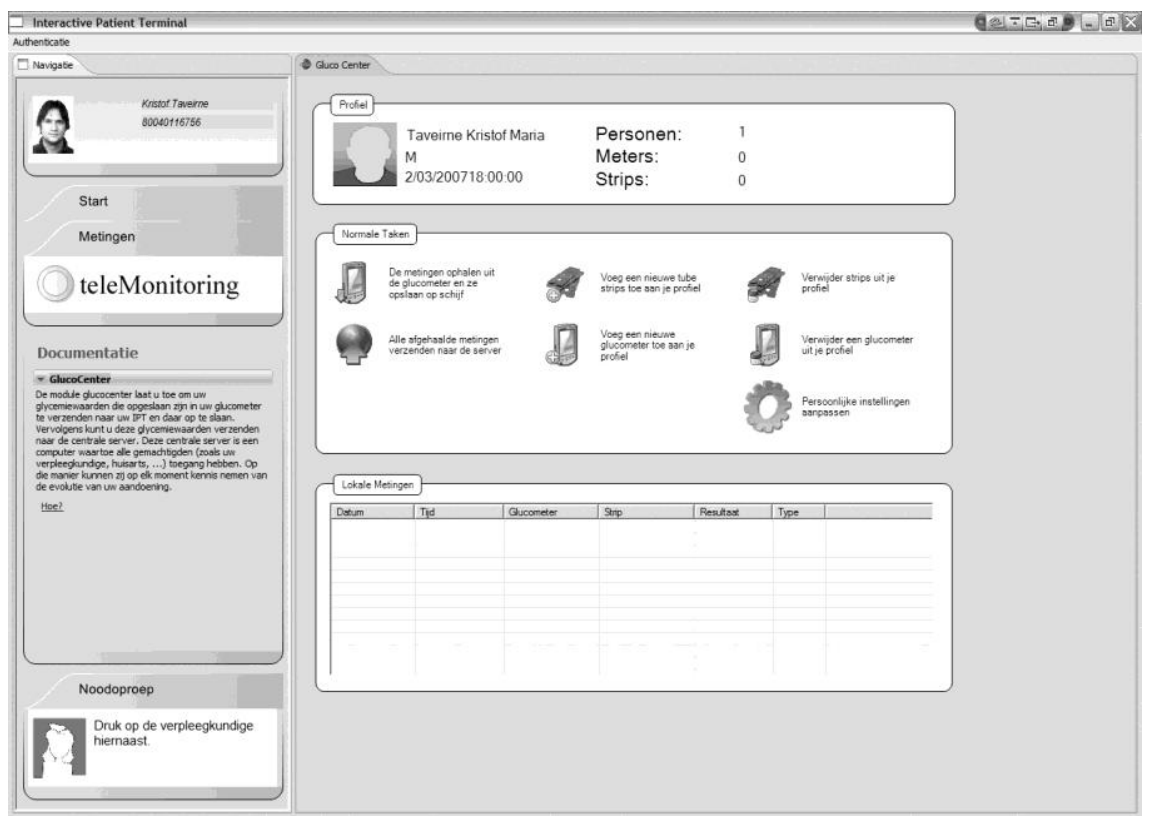

Figure 3: Client application with the tele-monitoring service activated. As the platform provides single-sign-on to the different services, the user is transparently authenticated to the tele-monitoring service.

Although a broker simplifies user actions and service provisioning, it complicates singlesign-on and requires delegation in order for the broker to take on the identity of the requester when executing a service request. For a more detailed description of the security architecture, the authors refer to [12].

\section{Results}

The impact of human factors on the use of technology is a fundamental issue, especially in the e-health domain. When technologies either appear difficult to use, are not adapted to the particular needs and thus perceived to be of little utility, they often are ignored. In order to evaluate the degree to which the e-health broker platform is usable, a platform prototype and the mentioned e-homecare services (e-scheduling, audio-diary, social games, video-chat, e-alarm, tele-monitoring) have been implemented within the Coplintho project [12], a project exploring the possibilities of using ICT in homecare.

Two complementary patient groups or pathologies were chosen in order to refine the focus of the demonstrator, namely diabetes and multiple sclerosis. Since several healthcare providers from different institutions are involved in the care process of diabetes patients, respectively multiple sclerosis patients, integration is needed and multidisciplinarity is guaranteed.

Figure 4 presents the testlab evaluation setting of the e-homecare broker platform, while Table 1 shows the average response times for starting and using the platform. 


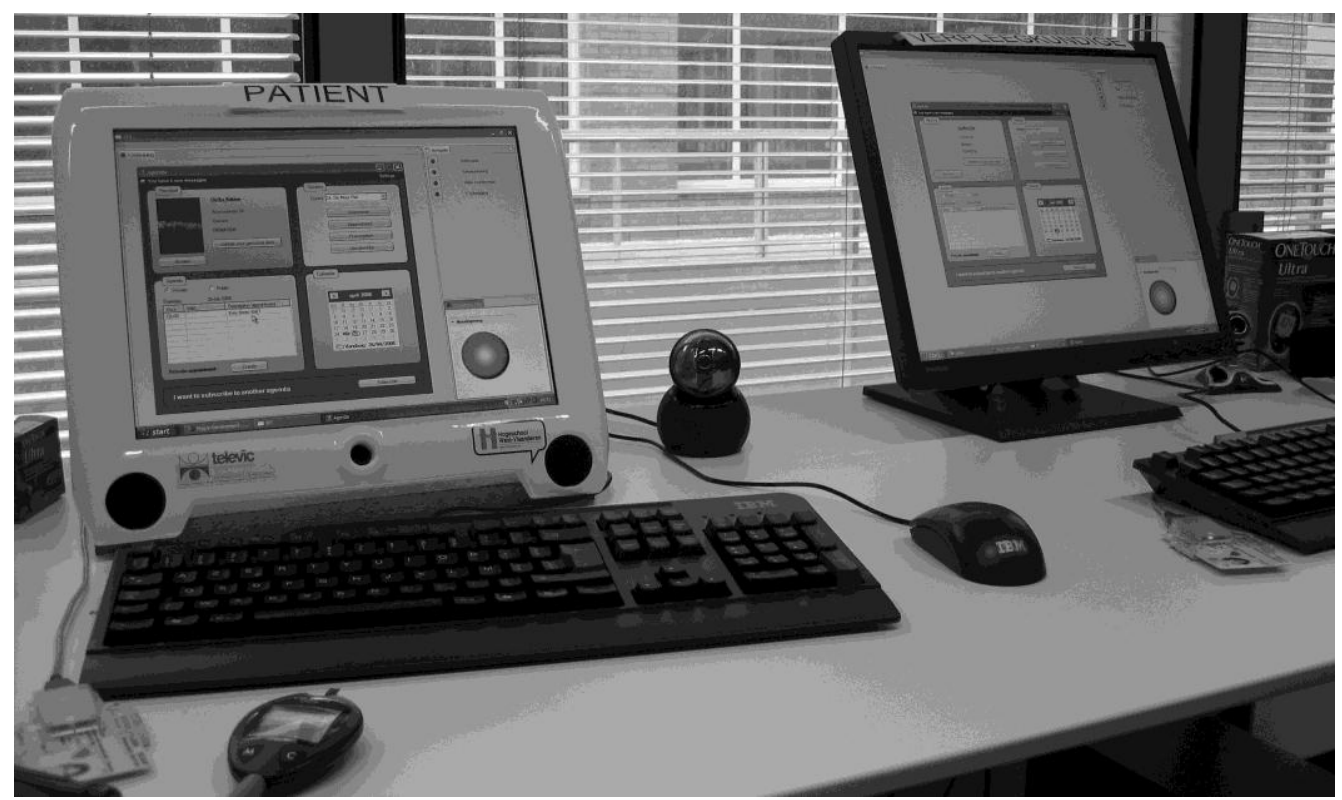

Figure 4: Testlab evaluation setup of the e-homecare broker platform.

\begin{tabular}{|l|c|}
\cline { 2 - 2 } \multicolumn{1}{c|}{} & Average time \\
\hline Single-sign-on login & $1 \mathrm{~s}$ \\
\hline Starting the client, logging in, downloading and installing the modules & $3 \mathrm{~s}$ \\
\hline Platform overhead & $135 \mathrm{~ms}$ \\
\hline
\end{tabular}

Table 1: Averages on startup time and execution overhead for the e-homecare platform.

As can be seen in the table, the total startup time was around 3 seconds for starting the client, logging in, downloading the modules, starting the modules and presenting the graphical interface. The total time spent in the broker platform in between receiving new patient data and presenting the output data in the client is around $135 \mathrm{~ms}$.

The startup time and overhead imposed by the platform was experienced by the patients to be small enough. The latter, combined with having all e-homecare services integrated in a single client application, requiring only one single login and being shielded from service management, resulted in a high quality of experience by the patients of using the e-homecare broker platform. The involvement of the medical experts, nurses and care providers in de development cycle of the e-homecare broker platform helped to establish greater user-acceptance by the patients.

\section{Discussion}

In order to save money and increase the patient's feelings of well-being at home, patients are discharged earlier and need to rely on e-homecare services monitoring their health and assisting them where needed. However, since not all patients are technical experts and e-homecare services are developed by different service providers, usability and acceptance of e-homecare might become difficult due to the complexity of managing all 
the different service locations and login information or due to unavailability of requested services.

Therefore the authors presented in this paper a client application and broker platform, minimizing user interactions and simplifying authentication, while providing dynamic service selection, security and data routing. The client application is adjusted dynamically to fit the patient profile, without any required interaction of the patient and fulfilling the specific needs for each patient personally by presenting only the e-homecare services that are of interest to the patient. Since each of the e-homecare service provider modules requires its own authentication, single-sign-on is used to alleviate users from authenticating to each service separately. The client application therefore interacts with the broker and delegates its right to access the e-homecare services to the broker using delegation assertions.

As the degree to which the e-health broker platform is usable either inhibits or facilitates its acceptance, use, and growth and its effectiveness as a solution for healthcare interoperability, insight was gained to determine how specific technological choices affect the usability. The evaluation showed a high quality of experience by the patients of using the e-homecare broker platform.

\section{Acknowledgements}

This work was partly funded by Transecare. Transecare is a project of IBBT (Interdisciplinary institute for BroadBand Technology). IBBT is a research institute founded by the Flemish Government in 2004.

\section{References}

1. Bloom DE, Canning D, Jamison DT. Health, Wealth and Welfare. Finance \& Development. 2004;31:10-15.

2. Institut national d'études démographiques (INED) [Internet] [cited 2009 Aug 31]. Available at: http://www.ined.fr.

3. Wartena F, Muskens J, Schmitt L. Continua: The Impact of a Personal Telehealth Ecosystem. In: Proceedings of IEEE eTelemed; 2009.

4. Lind L, Sundvall E, Karlsson D, Shahsavar N, Ahlfeldt H. Requirements and prototyping of a home health care application based on emerging JAVA technology. Int J Med Inform. 2002;68(1-3):129-139.

5. Van den Bosch B, Bellon E, De Deurwaerder A, Vanautgaerden M, Bangels M. Recommendations and quality criteria for hospital information systems. In: Federale Overheidsdienst Volksgezondheid .be Health Telematics; 2002.

6. OpenESB, the Open Source ESB for SOA \& Integration [Internet] [cited 2009 Aug 31]. Available at: https://open-esb.dev.java.net/.

7. Van Hoecke S, Taveirne K, De Turck F, Dhoedt B. Dynamic Selection of Interactive eHomeCare Services. In: Proceedings of the International Workshop on Services Integration in Pervasive Environments; 2007. 
8. Van Hoecke S, Taveirne K, Desmet S, Claerhout B, De Turck F, Dhoedt B. Secure and Dynamic Client Environment for Interactive eHomeCare. In: Proceedings of the 2007 International Conference on Software Engineering Research in Practice (SERP'07); 2007.

9. Van Hoecke S, Van Den Bossche B, Steurbaut K, Dhoedt B, Decruyenaere J, De Turck F. Effectively and efficiently routing medical decision support data in the Intensive Care Unit. Submitted to Computers in Biology and Medicine.

10. Van Hoecke S, Steurbaut K, Beheyt Y, De Bruyne F, Dhoedt B, Decruyenaere J, et al. Automatic WS-BPEL Composition of Medical Support Services in the ICU. In: Proceedings of the International Conference of eHealth, Telemedicine and Social Medicine; 2009.

11. McAffer J, Lemieux JM. Eclipse Rich Client Platform: Designing, Coding, and Packaging Java Applications. Addison-Wesley; 2005.

12. Ackaert A, Van Hoecke S, De Moor G, Spinhof L, De Rouck S, Agten S, et al. Innovative Communication Platforms for Interactive eHomeCare. In: Proceedings of the 5th IEEE Workshop on Applications and Services in Wireless Networks; 2005. 